\title{
Ueber das Verhalten des Sehpurpurs bei der Netzhautablösung.
}

\author{
Von \\ Dr. N. Andogsky \\ aus Petersburg.
}

(Ans dem Laboratorium von Prof. Th. Leber in Heidelberg.)

Zahlreiche und eingehende Untersuchungen von W.

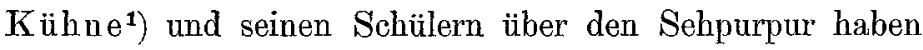
gezeigt, dass die Epithelschicht der Netzhaut, welche, wie bekannt, mit den den Sehpurpur enthaltenden Aussengliedern der Stäbchen in engerer Beziehung steht, als Regenerator des Sehpurpurs zu betrachten ist.

Untersuchungen über das Verhalten des Sehpurpurs bei Netzhautablösung, bei welcher diese engere Beziehung unterbrochen ist, da die Epithelschicht der Netzhaut auf der Oberfläche der Chorioidea haften bleibt, können nach zwei Richtungen hin von Interesse sein: 1. Zur Ausfüllung einer Lücke unserer Kenntnisse, über das Verhalten des Sehpurpurs bei einem nicht selten vorkommenden pathologischen Process der Netzhaut und 2. zur Controle der von W. Kühne aufgestellten Theorie.

1) Untersuchungen aus dem physiologischen Institute der Universität Heidelberg, Bd. I, II und III. Das Resumé seiner Arbeiten in Hermann's Handbuch der Physiologie, Bd. III, Th. 1. 1879: W. Kühne, Chemische Vorgänge in der Netzhaut. 
Ueber das Verhalten des Sehpurpurs bei der Netzhautablösung. 405

Bevor ich zur Mittheilung meiner eigenen Beobachtungen übergehe, erlaube ich mir hier in Kürze an die von W. Kühne erlangten Resultate zu erinnern.

Die unter gewissen Umständen zu beobachtende Purpurfärbung der Stäbchenaussenglieder der Netzhaut der meisten Thiere rührt von einem sie durchtränkenden lichtempfindlichen Stoffe her. Diese Färbung bleicht, dem Lichte ausgesetzt, bis zum völligen Verschwinden aus, und kann in einem normalen Auge bei Abschluss vom Lichte wieder zum Vorschein kommen. Der Process der Bleichung vollzieht sich gesetzmässig und stufenweise, wobei die Purpurfarbe allmählich in Roth, Rosa, Orange und Gelb übergeht, indem der Sehpurpur in Sehgelb - das erste ständige Product der Sehpurpurzersetzung übergeführt wird. Bei fortgesetzter Belichtung geht endlich das Sehgelb in das letzte Zersetzungsproduct des Sehpurpurs, das sogen. Sehweiss über. Bei Einwirkung des gewöhnlichen, gemischten Lichtes hängt die Schnelligkeit des Processes von der Intensität und Dauer der Belichtung ab. So bleicht z. B. die bei Sonnenlicht aus dem Auge eines Frosches herausgenommene Netzhaut in einigen Secunden, während sie bei schwachem Tageslicht einige Minuten, sogar 2030 Minuten, bis zum völligen Verschwinden der Färbung braucht.

Die Wirkung, welche Strahlen des Spectrums von gleicher Intensität, aber verschiedener Wellenlänge auf den Sehpurpur ausüben, ist verschieden. Die langwelligen Strahlen wie Roth und Gelb, führen den Sehpurpur langsamer in Sehgelb über als die Strahlen von kurzer Wellenlänge, vom violetten Ende des Spectrums; dagegen sind zu weiterer Ueberführung des Sehgelbs in Sehweiss die violetten Strahlen weniger wirksam, als die rothen und gelben.

Was den Process der Regeneration der im Dunkeln gehaltenen Netzhaut anlangt, so kam Kühne auf Grund verschiedener Experimente mit lebenden und aus dem Auge 
herausgenommenen Netzhäuten, theilweise auch mit Sehpurpurlösungen, zum Schluss, dass man hier eigentlich zwei Processe zu unterscheiden hat, welche von ihm als Anagenese und Neogenese bezeichnet wurden.

Der Process der Anagenese, welcher besonders deutlich an Kaltbliitern zu beobachten ist, besteht in der Regeneration des Sehpurpurs aus seinen Zersetzungsproducten mit Hilfe eines hypothetischen regenerirenden Stoffes, des Rhodophylin, welcher der Netzhaut durch ihr Epithel geliefert wird, wobei das letztere in lebendem oder wenigstens in überlebendem Zustand sich befinden muss. Mit Hilfe dieses Stoffes vollzieht sich die Regeneration schnell und mit derselben Gesetzmässigkeit wie die Zersetzung, aber in umgekehrter Reihenfolge.

Der Process der Neogenese besteht dagegen in der directen Erzeugung des Sehpurpurs durch die Epithelzellen oder in der Erreugung einer farblosen Substanz, welche sogleich in Purpur übergeführt wird, sobald sie an die Stäbchenschicht gelangt. Dieser Process vollzieht sich sehr langsam und lässt sich nur an lebenden Thieren beobachten.

Die Regeneration des Sehpurpurs erfolgt bald durch Anagenese, bald durch Neogenese, waș von verschiedenen Umständen abhängig ist. Bei Einwirkung sehr schwacher Beleuchtung auf das lebende Auge befinden sich die Zersetzung des Sehpurpurs und die Regeneration desselben aus den Zersetzungsproducten mit Hilfe der Anagenese im Gleichgewicht; eine schwache Belichtung kann daher keine Entfärbung der Netzhaut eines lebenden Auges hervorrufen. Bei stärkerer Belichtung prävalirt allmählich die Zersetzung über die Anagenese und wir bekommen eine allmähliche Bleichung des Purpurs. Wenn dabei nicht alle Zersetzungsproducte aus der Stäbchenschicht verschwunden sind, so kann bei Aufhebung der Belichtung die Färbung durch Uebergang dieser Producte in Sehpurpur wieder hergestellt 
Ueber das Verhalten des Sehpurpurs bei der Netzhautablösung. 407

werden. War aber das Auge sehr starker und andauernder Belichtung ausgesetzt, so wird der Purpur schnell in Sehweiss übergeführt, welcher wahrscheinlich durch Resorption aus der Stäbchenschicht entfernt wird. In diesem Falle reicht das Rhodophylin allein zur Herstellung der Netzhautfärbung nicht aus, da dasselbe in der Netzhaut kein Material zur Regeneration findet. Die Färbung kann nur durch die Uebertragung schon fertigen Purpurs aus dem Epithel der Netzhaut mit Hilfe des langsamen Processes der Neogenese wieder hergestellt werden.

Die im Laufe von 10 Minuten durch directes Sonnenlicht gebleichte Netzhaut eines lebenden Frosches zeigt bei nachträglichem Aufenthalt desselben im Dunkeln erst nach 20 Minuten die ersten Spuren der rosarothen Färbung und wird erst nach 1-2 Stunden vollkommen purpurn. Bei dem Kaninchen verläuft der Process etwas schneller. Nach 7 Minuten sind schon Spuren von Färbung wahrzunehmen und nach 35-38 Minuten völlige Regeneration. Bei gewöhnlicher, wechselnder Lichteinwirkung ron verschiedener Intensität können, wie Kühne annimmt, die Processe der Ana- und Neogenese zugleich stattfinden.

Die Netzhaut eines Dunkelthieres, welche demselben purpurn entnommen wurde, bleicht, an das Tageslicht gebracht, verhältnissmässig schneller als im Auge und kann sich unter gewissen Umständen wieder färben. Wird die isolirte, im Laufe von einigen Minuten gebleichte Froschnetzhaut wieder auf die Epithelschicht gebracht und im Dunkeln gehalten, so nimmt sie in 10-30 Minuten die Purpurfärbung wieder an, indem sie die oben erwähnten Stadien der Färbung durchmacht. In diesem Falle findet der Process der Anagenese statt. Wie Kühne annimmt, bleiben in der isolirten gebleichten Netzhaut alle Zersetzungsproducte des Purpurs in den Stäbchen haften, da die Entfernung derselben nur durch Lymphströmungen möglich ist. Das zur Regeneration dieser Producte nöthige 
Rhodophylin kann der Netzhaut durch die Berührung mit dem Epithel zugeführt werden, da sich dieses im Stadium des Ueberlebens befindet, welches bei Kaltbliutern ziemlich lange dauert. Fuir die Anagenese spricht in diesem Falle. auch die kurze Zeitdauer, welche für die Wiederherstellung der Färbung nöthig ist (10-30 Minuten), während die Neogenese sogar im lebenden Auge des Frosches bedeutend langsamer verläuft (1-2 Stunden). Der Versuch lässt sich ein bis zwei Mal wiederholen, aber mit geringerem Effect. Auch an einer isolirt gebleichten und in's Dunkle gebrachten Froschnetzhaut, welche nicht mit dem Epithel in Berührung gebracht worden ist, findet Regeneration des Sehpurpurs statt; dieselbe verläuft aber bedeutend langsamer, als mit Hilfe des Epithels (sie erfordert 2-3 Stunden), und ist nicht so ausgesprochen. Dieser Process wird von Kühne als Autoregeneration bezeichnet. Kühne nimmt an, dass selbst wenn man die Netzhaut vom Epithel vollständig abzieht, der Stäbchenschicht noch Epithelreste oder kleine Mengen gelösten, frïher gelieferten Rhodophylins anhaften bleiben, welche fähig sind, die Zersetzungsproducte des Sehpurpurs wieder zur Regeneration zu bringen.

Was nun die Regenerationsprocesse an isolirten Netzhäuten der Säugethiere anlangt, so sind dieselben kaum nachweisbar. Wenn man eine isolirt gebleichte Kaninchennetzhaut auf kurze Zeit mit der Epithelschicht in Berührung bringt, so tritt keine Wiederfärbung der Netzhaut ein. Die Ursache ist in der Unmöglichkeit der Neogenese zu suchen, da das Auge abstirbt; die Anagenese kann hier auch nicht in dem Maasse zur Geltung kommen, wie bei dem Frosche, da bei den Säugethieren ein solcher Zustand des Ueberlebens, dass das Epithel nach dem Tode noch Rhodophylin $\mathrm{zu}$ produciren im Stande ist, zu kurze Zeit dauert und fast nicht nachweisbar ist. Nur sehr schwache Autoregeneration konnte $\mathrm{K} \ddot{u} \mathrm{hne}$ an isolirt gebleichten Kaninchen- 
Ueber das Verhalten des Sehpurpurs bei der Netzhautablösung. 409

netzhäuten beobachten, wenn diese, obschon ohne Berührung mit dem Epithel, sehr lange Zeit (2-3-24 Stunden) im Dunkeln verblieben. Er giebt für diese Erscheinung dieselbe Erklärung, wie bei der Autoregeneration der Froschnetzhaut.

Wie aus obigen Mittheilungen hervorgeht, kann man den Regenerationsprocess der isolirt gebleichten Netzhaut nur dann auf Anagenese zurückführen, wenn man annimmt, dass nicht alle Zersetzungsproducte der Netzhaut entfernt wurden. Die Richtigkeit dieser Voraussetzung zeigt folgender Versuch: wenn man einem Frosch die Netzhaut entnimmt, nachdem sie ihren Purpur im lebenden Zustande des Thieres verloren hat, so zeigt sich keine Wiederherstellung der Farbe, auch wenn die Netzhaut längere Zeit auf der Oberfläche der Epithelschicht im Dunklen gehalten wird; es ist also augenscheinlich, dass bei völliger Bleichung der Netzhaut im lebenden Thiere die Zersetzungsproducte des Purpurs sofort aus der Stäbchenschicht verschwinden.

Vollzieht sich die Regeneration des Sehpurpurs bald durch den vitalen Process der Neogenese, bald durch den rein chemischen Process der Anagenese, - so spielt jedenfalls die Epithelschicht der Retina eine sehr wichtige Rolle, indem sie in dem einen Falle den Purpur liefert, in dem anderen das Rhodophylin producirt, einen Stoff, welcher den Sehpurpur aus seinen Zersetzungsproducten zu regeneriren vermag.

Im lebenden Auge vollzieht sich der Process der Regeneration unabhängig von irgend welchem Nerveneinfluss; derselbe verändert sich nicht nach Durchschneidung des N. opticus, N. trigeminus, N. oculomotorius, N. sympathicus; Vergiftung mit Curare und Atropin hat bei Säugethieren keinen Einfluss auf den Gang des Processes. Nur Veränderungen des Kreislaufs und der Lymphströmungen sind auf die. Schnelligkeit der Regeneration von Einfluss. So lässt sich eine Verlangsamung des Processes bei erhöhtem 
intraocularem Druck und bei vermindertem Blutzufluss zum Auge nach starken Blutentziehungen beobachten. Im Gegentheil kann man durch Pilocarpin und Muscarin den Regenerationsprocess beschleunigen; da diese Gifte uiberhaupt die Secretionen beschleunigen, so nimmt $K \ddot{u} h n e$ an, dass sie auch hier die vermuthliche Secretion des Netzhautepithels verstärken.

Aus obigen Mittheilungen ergiebt sich, von welchem Interesse es ist, das Schicksal des Sehpurpurs bei Netzhautablösung, wo die noch lebende Netzhaut nicht mehr mit ihrem Epithel in Berührung steht, zu verfolgen. Besonders wichtig ist diese Untersuchung bei Säugethieren, bei welchen wegen der Unfähigkeit der Gewebe zum Ueberleben der Versuch mit der exstirpirten Netzhaut über den Gang des Processes im Leben keinen Aufschlúss geben kann.

In der Literatur dieser Frage finden sich fast keine experimentellen Untersuchungen darüber. Einiges darauf beziigliche findet sich in einer Arbeit von Ewald und Kühne ${ }^{1}$ ).

Um die Berührung des Netzhautepithels mit der Stäbchenschicht bei lebenden Fröschen zu unterbrechen, curarisirten Ewald und Kühne dieselben und hielten sie dann in warmem Wasser. Der sich in Folge dessen entwickelnde allgemeine ödematöse Zustand betrifft auch das Auge und. ruft durch die Durchtränkung der Gewebe mit Flüssigkeit eine Trennung des Epithels der Netzhaut von der Stäbchenschicht hervor. Wenn das Thier bis vor dem Experiment und nach demselben im Dunkeln gehalten wurde, so bleibt die Netzhaut in den ersten Tagen intensiv purpurn; mit der Zeit aber wird die Purpurfarbe allmählich schwächer, so dass nach mehreren Tagen nur schwache Rosafarbe der Netzhaut zu constatiren ist. Um diese allmähliche Bleichung

1) A. Ewald und W. Kühne, Untersuchungen über den Sehpurpur. Untersuch. aus d. Phys. Inst. d. Univ. Heidelb. Bd. I. H. 3. S. 254, Bd. I. H. 4, S. 371. 
Ueber das Verhalten des Sehpurpurs bei der Netzhautablösung. 411

der Färbung ohne Lichtzufluss zu erklären, nehmen die Verfasser an, dass hier eine langsame Resorption des Sehpurpurs durch Lymphströmungen stattfinde. Wenn man einen curarisirten Dunkelfrosch starker Belichtung aussetzt, so ist die Netzhaut, nachdem sie in gebleichtem Zustande dem Thiere entnommen wurde, sowohl zur Regeneration, als zur Autoregeneration vollkommen unfähig, so wie auch jede intra vitam gebleichte und daher der Zersetzungsproducte ermangelnde Netzhaut.

Th. Leber ${ }^{1}$ ) theilt bei der Discussion eines Vortrages von $O$. Becker über die Anatomie der Netzhautablösung mit, dass auf seine Veranlassung Nordenson schon einige Versuche über das Verhalten des Sehpurpurs bei experimentell erzeugter Netzhautablösung angestellt habe. Es wurde kein Sehpurpur gefunden, was aber darauf beruhte, dass die Stäbchenschicht schon zu sehr degenerirt war, so dass sich aus diesen Versuchen keine Schlüsse ziehen liessen.

In der Abhandlung von $\mathrm{H}$. Adler ${ }^{2}$ ): „Beobachtungen über das Vorkommen von Sehpurpur am kranken und verletzten Menschenauge" ist folgender Fall beschrieben:

Bei einem 7 Jahre alten Mädchen wurde das linke Auge mit einem Taschenmesser stark verletzt. Sofort nach der Verletzung wurde von der Mutter des Kindes Verband angelegt, und $1 / 4$ Stunde später hatte Adler die Gelegenheit, das Auge zu untersuchen. Er fand eine grosse verticale Corneawunde, ebenso die Iris zerschnitten. Nach innen hinter der Pupillarebene lagerte ein Stück abgerissener Netzhaut auf einem nach innen unten dislocirten Linsenfiagmente. Dasselbe war dunkelroth gefärbt, wobei diese Farbe ganz dentlich neben den gut injicirten Gefässen hervortrat. Die Beleuchtung mit einer Petroleumlampe änderte diese Farbe fast gar nicht, nur nach $3 / 4$ stündigem Aufenthalt beim Tageslicht schwand dieselbe allmählich, aber anch nicht vollständig. Es wurde wieder ein Druckverband angelegt. Fünf Stunden später fand sich beim Abnehmen des

1) Bericht über die XX. Versammlung der Ophthalmologischen Gèsellschaft zu Heidelberg, 1889, S. 127-128.

2) Centralblatt f. die med. Wissenschaften, 1877, Nr. 14, S. 242. v. Graefe's Archiv für Ophthalmologie. XLIV. 
Verbandes die Röthung der Netzhaut vollkommen versehwunden und kehrte auch später nicht mehr wieder. Der Verfasser zieht aus diesem Falle Schlüsse über das Verhalten des Sehpurpurs an der von der Chorioidea abgetrennten Retina, und zwar 1. dass der Sehpurpur sich längere Zeit, mindestens eine Stunde lang, bei mehrfacher Belichtung durch Tageslicht erhalte, und 2. dass der einmal verschwundene Sehpurpur einer abgelösten Parthie trotz des Zusammenhanges derselben mit der übrigen Netzhaut und des Fortbestandes eines gewissen Grades von Ernährung und trotz des absoluten Schutzes vor eindringendem Lichte, nicht wieder hergestellt werden könne.

Diesen Schlüssen ist aber, soweit sie mit sonst bekannten Erfahrungen im Widerspruch stehen, durchaus nicht zuzustimmen, da man doch nicht jedes rothe Aussehen einer abgelösten Netzhaut auf Sehpurpur beziehen darf. Die dunkelrothe Färbung, welehe der menschliche Sehpurpur niemals besitzt, zeigt vielmehr, dass es sich wohl nur um eine Blutung gehandelt hat.

Das Fehlen weiterer experimenteller Untersuchungen über die aufgeworfene Frage, obgleich seit der Entdeckung der lichtempfindlichen Purpurfärbung der Netzhaut durch Boll 20 Jahre verflossen sind, zeigt hinlänglich, dass das rege Interesse, welches diese Entdeckung hervorgerufen hatte, bei den Ophthalmologen bald erloschen ist. Die Experimente W. Kühne's an Thieren und die Untersuchungen über die Verbreitung des Sehpurpurs im Thierreiche haben gelehrt, dass das Sehvermögen erhalten bleibt, auch wenn der Sehpurpur durch Belichtung vollkommen gebleicht ist, und dass es Thierarten giebt, deren Netzhaut keine Stäbchen, also auch keinen Sehpurpur enthält; man kann daher die Zersetzung des Sehpurpurs durch das Licht nicht als unentbehrliches Erforderniss für den Process des Sehens betrachten. Auch der Urheber der photochemischen Theorie des Sehens, W. Kühne, betrachtet den Sehpurpur nur als einen von den vielen vorausgesetzten Sehstoffen, deren photochemische Veränderungen dem Sehact zu Grunde liegen. 
Ueber das Verhalten des Sehpurpurs bei der Netzhautablösung. 413

In der letzten Zeit ist, wie bekannt, die Frage über den Sehpurpur, wie auch über die Stoffe, welche in engerer Beziehung zu der Farbenempfindung stehen, wieder in der Iiteratur aufgetaucht. A. König ${ }^{1}$ ) stellte die Hypothese auf, dass eine schwache Zersetzung des Sehpurpurs die Empfindung Grau hervorrufe, während bei stärkerer Lichteinwirkung, welche nicht nur den Sehpurpur in Sehgelb. überführt, sondern auch das letztere zur Zersetzung bringt, - Blauempfindung entstehen soll. Da die Fovea centralis nur Zapfen enthält und in Folge dessen des Sehpurpurs entbehrt, so würde hieraus folgen, dass die Fovea blaublind sein muss. A. König hat in der That diese Folgerung gezogen und behauptet die Blaublindheit der Fovea, gestïtzt auf gewisse Beobachtungen, deren Beweiskraft aber durch die leicht $\mathrm{zu}$ constatirende Thatsache widerlegt wird, dass man mit dem der Fovea entsprechenden centralen Theil des Gesichtsfeldes Blau ganz gut sehen kann, wie E. Hering ${ }^{2}$ ) and W. Koster ${ }^{3}$ ) hervorgehoben haben. Hiernach ist die Theorie von A. König, dass die Blauempfindung durch die Zersetzung von Sehgelb zu Sehweiss erzeugt werde, wenig wahrscheinlich, während vieles für die Annahme von v. Kries ${ }^{4}$ ) spricht, wonach die den Sehpurpur enthaltenden Stäbchen nur eine farblose Lichtempfindung vermitteln. Bekanntlich tritt bei Netzhautablösung, wie Th. Leber ${ }^{5}$ ) zuerst berichtet hat, nicht selten eine Störung

1) A. König, Ueber den menschlichen Sehpurpur und seine Bedeutung für das Sehen. Sitzungsberichte der königl. Academie der Wissenschaften zu Berlin. 1894. S. 577.

2) E. Hering, Ueber angebliche Blaublindheit der Fovea centralis. Pflüger's Archiv, LIX. S. 403-414 (1894).

3) W. Koster, Untersuchungen zur Lehre vom Farbensinn. v. Graefe's Archiv f. Ophthalm. XII. 4. S. 1-20. (1894).

4) v. Kries, Deber die Function der Netzhautstäbchen, Zeitschrift f. Psych. u. Physiol. der Sinnesorgane. IX. S. 81-123 (1895).

5) Th. Leber, Krankheiten der Netzhaut und des Sehnerven in Graefe-Saemisch's Handbuch. V. S. 690 (1877). 
des Farbensinnes auf, bei welcher Blau mit Grün verwechselt wird; dieselbe wurde auch bei manchen Formen von Retinitis gefunden, unter anderen von R. Simon ${ }^{1}$ ) in mehreren Fällen von Retinitis albuminurica, aber beschränkt auf die nächste Umgebung des Fixirpunktes, und wurde hier von A. König auf Violettblindheit bezogen. Th. Leber ist geneigt, diese Störung durch eine gelbe Farbe der subretinalen Flïssigkeit zu erklären; Simon glaubt aber für seine Fälle eine Erklärung durch eine dioptrische Störung nicht annehmen zu können und führt sie auf „Functionsunfähigkeit der blauempfindenden photochemischen Substanz" zurück, die er aber nicht mit dem Sehpurpur für identisch erklärt. Von letzterem kann auch nicht die Rede sein, da die Störung auf das des Purpurs entbehrende Netzhautcentrum beschränkt war.

Bei der Ausführung der vorliegenden Arbeit, welche auf Anregung des Herrn Prof. Th. Leber unternommen wurde, habe ich nicht im Auge gehabt, den Process der Regeneration und der Zersetzung des Sehpurpurs in Beziehung auf Licht- und Farbenempfindungen zu untersuchen, sondern auf experimentellem Wege festzustellen, ob in der abgelösten Netzhaut Purpur zu beobachten ist, und sein weiteres Schicksal zu verfolgen.

Um eine experimentelle Netzhautablösung hervorzurufen, stehen uns bisher als Methoden nur die Einführung von Fremdkörpern, besonders aus Metall, und die Injection von Flüssigkeiten, welche eine Schrumpfung des Glaskörpers bewirken, zu Gebote. Dem Mechanismus der Netzhautablösung nach scheinen diese Methoden auch die zweckmässigsten zu sein, da, wie die Untersuchungen ron

1) R. Simon, Ueber typische Violettblindheit bei Retinitis albuminurica. Centralblatt f. prakt. Augenh. 1894. S. 132. 
Ueber das Verhalten des Sehpurpurs bei der Netzhautablösung, 415

Th. Leber ${ }^{1}$ ) und E. Nordenson ${ }^{2}$ ) bewiesen haben, die Glaskörperschrumpfung auch bei der Netzhautablösung im Menschenauge die Hauptrolle spielt. Durch welche Methode aber auch die experimentelle Ablösung der Netzhaut hervorgerufen sein mag, stets kommt es zu einer sehr raschen Degeneration der abgelösten Membran, bald in allen ihren Schichten, bald nur in der Stäbchenschicht, welche letztere bei der uns interessirenden Frage ja vorzugsweise in Betracht kommt. Hierbei kann ich jedoch nicht unterlassen, zu bemerken, dass die an der abgelösten Thiernetzhaut gemachten Beobachtungen nicht vollständig mit den Erfahrungen am menschlichen Auge übereinstimmen.

Nachdem ich zu dem genannten Zweck eine ganze Reihe von Substanzen in den Glaskörper einzuführen versucht hatte, fand ich schliesslich die Einführung von Fremdkörpern aus Kupfer und die Injection von physiologischer Kocbsalzlösung in den Glaskörper unter erhöhtem Drucke als die zweckmässigsten, am sichersten zum Ziele führenden Methoden, welche trotz der sich schnell vollziehenden Veränderungen an der Netzhaut das Verhalten des Sehpurpurs zu beobachten gestatten.

Die Versuche mit Fremdkörpern aus Kupfer wurden sämmtlich am Kaninchenauge folgendermaassen ausgeführt: Ein $1-11_{2} \mathrm{~mm}$ dickes, $5 \mathrm{~mm}$ langes Stück Kupfer wurde unter aseptischen Kautelen durch eine kleine Skleralwunde in der Mitte zwischen dem Eintritt des N. opticus und dem Limbus corneae bei luxirtem Auge in den Glaskörperraum eingeführt. Nach dieser Operation zeigten die Augen gewöhnlich keine Reizerscheinungen und die kleine Wunde heilte sehr schnell von selbst. Die Fremdkörper blieben

5) Th. Leber, Ueber die Entstehung der Netzhautablösung. Bericht über die XIV. Versammlung d. -ophthalm. Gesellschaft zu Heidelberg. Klin. Monatsbl. 1882. Beilageheft, S. 18. - Derselbe, Die Entstehung der Entzündung etc. 1891, S. 229.

2) E. Nordenson, Die Netzhautablösung. Wiesbaden 1887. 
manchmal oben innerhalb der Wunde stecken, aber öfters senkten sie sich und kamen auf den seitlichen oder unteren Theil der Netzhaut, unweit des Ciliarkörpers zu liegen. Nach anderthalb bis zwei Tagen ist der Fremdkörper gewöhnlich mit Exsudat bedeckt, und in seiner unmittelbaren Nähe bildet sich eine dichte Trübung des Glaskörpers aus, von welcher nach allen Richtungen hin feine Züge ausstrahlen. Die Netzhaut selbst wird schon nach ein bis zwei Tagen an mehreren Stellen mit einer feinen Exsudatschicht bedeckt. In den Fällen, in denen das Kupferstück zwar aseptisch war, aber auf seiner Oberfläche Spuren von Oxyd aufwies, entwickelten sich auf der Oberfläche der Netzhaut und in dem Glaskörper sehr starke Trübungen, der ganze Process verlief stürmischer, und ging mit stärkerer Entzündung und Degeneration der Augenhäute einher. Die Netzhautablösung wurde gewöhnlich drei bis zehn Tage nach Einführung des Fremdkörpers bemerkbar, öfters aber erst nach vier bis fünf Tagen, wobei die Ablösung eines beträchtlichen Theiles derselben auf einmal ziemlich schnell (innerhalb 12-24 Stunden) erfolgte. Eine directe Beziehung zwischen der Lage des Fremdkörpers und der Stelle der Ablösung der Netzhaut liess sich nicht nachweisen. Manchmal fand eine Ablösung des dem Fremdkörper gegenüber liegenden Theiles der Netzhaut statt, manchmal des daneben liegenden Theiles derselben; öfters löste sich eine Hälfte der Netzhaut, die innere, äussere, obere oder hintere ab, manchmal aber beschränkte sich die Ablösung auf die markhaltige Netzhaut und der ihr unmittelbar anliegenden Theile der marklosen; schliesslich erfolgte manchmal eine totale Ablösung. In den meisten Fällen wurden in der Netzhaut Risse beobachtet, gewöhnlich in horizontaler Richtung, ober- und unterhalb der Markflügel, wobei sie entweder der Ablösung vorausgingen oder während derselben entstanden. In seltenen Fällen entstanden die Risse an der Ora serrata neben dem Fremdkörper, welcher, nach der 
Ueber das Verhalten des Sehpurpurs bei der Netzhautablösung. 417

Ablösung der anliegenden Theile, zwischen der Netzhaut und der Chorioidea sich befand.

In den meisten Versuchen wurden die Augenhäute nach Beendigung der Untersuchung auf Sehpurpur auch der mikroskopischen Untersuchung unterzogen, theils um im Allgemeinen die Veränderungen festzustellen, welche die Netzhaut durch die Wirkung des Fremdkörpers erfahren hatte, theils und insbesondere um $\mathrm{zu}$ ermitteln, wie weit die Stäbchenschicht sich dabei noch gut erhalten hatte, da bei einer weit gediehenen Zerstörung derselben natürlich auch kein Sehpurpur mehr erwartet werden kann.

Die pathologisch-anatomischen Veränderungen des Auges unter Einwirkung von Fremdkörpern aus Kupfer sind schon ausführlich von Th. Leber ${ }^{1}$ ) beschrieben worden; die Frgebnisse unserer Versuche stimmen im Allgemeinen und Wesentlichem mit dieser Beschreibung, was die Retina und Chorioidea anbetriffit, vollkommen überein.

Der dem Fremdkörper unmittelbar anliegende Theil der Netzhaut war in der Regel eitrig infiltrirt und nekrotisirt. In Bezug auf die Veränderungen der übrigen Netzhaut lässt sich kein allgemeines Schema aufstellen. Die entzündlichen und degenerativen Processe waren bald an dem abgelösten Theil der Netzhaut mehr ausgesprochen, wobei der unabgelöste ziemlich gut erhalten blieb, bald, was aber seltener zur Beobachtung kam, war der nicht abgelöste Theil vollständig zerstört, während der abgelöste ziemlich gut erhalten geblieben war; der Grad der Degeneration der abgelösten Theile entsprach auch im Allgemeinen der Dauer der Ablösung nicht vollkommen.

Von den pathologisch-anatomischen Veränderungen der abgelösten Parthieen sind diejenigen der Stäbchenschicht für uns von besonderem Interesse. Diese Veränderungen bestanden in varicöser Degeneration der Stäbchen, welche

1) Th. Leber, Die Entstehung d. Entzünd. 1891. S. 259-271. 
in der Mehrzahl der Fälle zu beobachten war; dabei waren in einigen Fällen nur die äusseren Glieder der Stäbchen, in anderen auch die inneren degenerirt, so dass man die Grenze zwischen diesen Gliedern nicht unterscheiden konnte. Als weitere Stadien der Degeneration wurden auch Ersetzung der Stäbchenschicht durch ziemlich grosse Eiweisstropfen oder Zusammenfliessen der Stäbchen in eine structurlose Masse beobachtet. Nicht selten wurde diese Schicht auch von Wanderzellen durchsetzt. In manchen Fällen, welche den späteren Stadien der Netzhautablösung entsprachen, fehlte die Stäbchenschicht und Degenerationsproducte derselben vollständig.

Veränderungen des Pigmentepithels') kamen auch fast in allen Fällen vor und bestanden in einer unregelmässigen Vertheilung des Pigments in den Epithelzellen, oder in vollständigem Verschwinden des Pigments aus einzelnen Zellen; manchmal waren auch ausgedehnte Wucherungen des Epithels zu beobachten. Die eiweisshaltige Flüssigkeit, welche zwischen der Retina und der Chorioidea sich befand, enthielt fast immer eine mehr oder weniger reichliche Beimengung von Zellenelementen, wobei sich dieselben besonders an der Oberfläche des Pigmentepithels anhäuften. Ausser kleinen vielkernigen Elementen fanden sich noch in ziemlich reichlicher Menge grosse Pigmentkörnchen- und Fettkörnchenzellen.

Was die Veränderungen der anderen Schichten der abgelösten Parthieen der Retina anbelangt, so waren sie in verschiedenen Fällen verschieden ausgesprochen. Die äussere Körnerschicht zeigte ausser einer gewissen ödematösen Auflockerung ihrer Elemente oft ein eigenthümliches Bild durch ihr zackiges Hineinragen in die Stäbchenschicht. Die reticulären Schichten hatten manchmal eine gröbere Structur, was auch auf Oedem der Retina beruhte. Die

1) Für die Versuche wurden nur Kaninchen mit pigmenthaltigen Augen benutzt. 
Elemente der inneren Körnerschicht zeigten Auflockerung; und in einigen Fällen waren, dieser Schicht entsprechend, Längsrisse der Netzhaut zu sehen. Die Ganglienzellenund die Nervenfaserschicht erfuhren seltener Veränderungen als die anderen Schichten, doch waren auch hier in manchen Fällen ein körniger Zerfall des Protoplasmas der Ganglienzellen, völliger Schwund der Zellen und sogar völlige Zerstörung beider Schichten zu beobachten. In allen Retinaschichten konnte man auch sehr oft eine Durchsetzung mit Wanderzellen constatiren.

Die nicht abgelösten Theile der Netzhaut blieben in der Mehrzahl der Fälle ziemlich gut erhalten; es war auch hier manchmal eine beginnende varicöse Degeneration der äusseren Glieder der Stäbchen zu beobachten, und in seltenen Fällen war eine vollständige Frweichung und Zerstörung aller Schichten vorhanden. Veränderungen der Chorioidea wurden nur verhältnissmässig selten beobachtet und bestanden in Erweiterung der Gefässe und Vermehrung der zelligen Elemente.

Die andere Methode, um Netzhautablösung hervorzurufen, bestand in der Einspritzung von sterilisirter physiologischer Kochsalzlösung in den Glaskörper unter erhöhtem Druck. Ich benutzte dazu einen sehr einfachen Apparat, nämlich einen Trichter, welcher durch einen Kautschukschlauch mit einer ziemlich dicken Stichcanüle verbunden war. Der Apparat wurde mit sterilisirter $3 / 4 \% \mathrm{NaCl}$ Lösung gefüllt und so aufgestellt, dass das Niveau der im Trichter befindlichen Lösung $50-55 \mathrm{~cm}$ über dem Kaninchenauge stand. Nachdem die Caniile in den Glaskörper eingeführt war, wurde die Flüssigkeit unter einem Drucke, welcher etwa dem von $40 \mathrm{~mm} \mathrm{Hg}$ entsprach, in das Auge einfliessen gelassen. Nach mehrmaliger Punction der Kammer konnte man auf diese Weise in Verlaufe von $3 / 4$ bis 1 Stunde einige Cubikcentimeter Flüssigkeit in das Auge einführen. 
Die Ablösung der Netzhaut erfolgte hiernach in einigen Augen nach fünf bis sechs Tagen, wobei dieselbe auch bei dieser Methode der Ablösung wider Erwarten Veränderungen ihrer Structur zeigte und bis zum neunten Tage nach dem Beginn der Ablösung vollständig structurlos wurde.

Eine Veränderung in der Anstellung dieses Versuches ermöglichte, eine raschere Ablösung der Netzhaut herbeizuführen, wenn auch mit noch stärkeren Veränderungen der Augenhäute.

Dieselbe besteht darin, dass man gleichzeitig bei der Einführung der Flüssigkeit in den Glaskörper auf der entgegengesetzten Seite der Sklera eine kleine Wunde anbringt. Dies ermöglicht eine schnellere Circulation der Flüssigkeit im Auge, so dass im Laufe von ${ }^{3} / 4$ bis 1 Stunde durch dasselbe $200 \mathrm{cbcm}$ und noch mehr durchfliessen können. Eine solche Ausspülung des Glaskörpers führt ziemlich schnell zur Schrumpfung desselben, so dass in einigen Fällen schon am Schlusse des Versuches die beginnende Netzhautablösung ophthalmoskopisch zu beobachten ist. Einige Stunden nach der Einspritzung der Flüssigkeit entwickelt sich eine dichte Trübung des Glaskörpers, welche die ophthalmoskopische Untersuchung erschwert.

Die Controlversuche, welche an den Augen 2 bis 24 Stunden nach einer solchen Injection unternommen wurden, zeigten, dass eine partielle, öfter aber eine totale Netzhautablösung gleich nach der Einführung der Flüssigkeit oder an demselben Tage erfolgte. Der Glaskörper schrumpfte in solchen Augen zu einer fibrinhaltigen Masse zusammen und zog die Falten oder Risse zeigende Netzhaut, welche an dem Glaskörper anliegen blieb, mit sich.

Was die Veränderungen der Netzhaut anbelangt, so sind sie bei dieser Methode denjenigen bei der Einführung von Fremdkörpern im Allgemeinen ähnlich und bestehen in entziindlichem Oedem mit mehr oder weniger ausgesprochener Durchsetzung mit Wanderzellen und in Degeneration 
Ueber das Verhalten des Sehpurpurs bei der Netzhautablösung. 421

der Netzhautelemente. Sie entwickeln sich aber hier mehr allmählich und proportional der Dauer der Ablösung. Die uns besonders interessirende Stäbohenschicht ist am ersten und sogar am zweiten Tage nach der Entstehung der Ablösung noch ziemlich gut erhalten, am dritten Tage ist schon eine ausgesprochene varicöse Degeneration und stellenweise auch Abhebung von anderen Schichten zu beobachten; am sechsten Tage sind nur Reste der degenerirten Schicht hier und da zu sehen und später schwinden die Stäbchen vollständig.

Wie aus der Beschreibung dieser Methoden der Hervorrufung der Netzhautablösung zu sehen ist, sind dieselben nicht ganz vollkommen, da sie starke Veränderungen der Netzhaut hervorrufen. Aber die anderen versuchten Methoden (Einführung von verschiedenen sonstigen metallischen und nichtmetallischen Fremdkörpern, Injection von Oxalatplasma, löslicher Kieselsäure '), Sublimatlösung u. s. w.) stellten sich als weniger zuverlässig heraus, oder riefen in der Netzhaut dieselben, wenn nicht noch stärkere Entzündungs- und Degenerationsvorgänge hervor.

Da ich auf die Einzelheiten der Anordnung der Versuche bei der Beschreibung jeder Gruppe derselben näher eingehe, so brauche ich an dieser Stelle nur einiges über die Methodik der Untersuchung im Allgemeinen zu sagen.

Um die schädliche Wirkung des Lichtes auf die Augen, wo Sehpurpur zu erwarten war, zu vermeiden, wurden alle Manipulationen mit denselben im dunkeln Zimmer bei Natronlicht vorgenommen. Um dasselbe zu erzeugen, wurde in die Flamme eines Bunsen'schen Brenners ein auf einem Platindraht angeschmolzenes Sodastiickchen eingeführt. Diese von W. Kühne angegebene Beleuchtungsmethode ist bei

1) Ich verdanke diese Substanzen der Güte von Herm Geheimrath $W$. Kühne. 
gewöhnlicher Intensität für den Sehpurpur sogar bei stundenlanger Arbeit indifferent. Bei derselben Beleuchtung wurden auch die Einspritzungen der $3 / 4 \%$ NaCl-Lösung gemacht, da die Erfahrung zeigte, dass die Ablösung nicht selten schom bei der Einspritzung entstand.

Die zu untersuchenden Kaninchenaugen wurden gewöhnlich bei Natronlicht enucleirt ${ }^{1}$ ) und entweder im frischem Zustande, oder, was öfters vorkam, nach 3-24stündiger Härtung, im Dunkeln in $10 \%$ Formollösung aufgeschnitten. Dieses Formolverfahren, welches jetzt die früher von $W . K$ ühne angegebene Alannisirung ersetzt, hat den Zweck, die Netzhaut zu härten, um dieselbe leichter ohne Zerreissung und Lockerung ihrer Gewebe von der Chorioidea abziehen zu können. Dabei schadet die Formollösung dem Purpur gar nicht, im Gegentheil sie macht denselben mehr resistent gegen die Lichteinwirkung.

Die Technik der Präparation des Auges war folgende: das Auge wird gewöhnlich äquatorial halbirt und der Glaskörper mittelst einer Pincette entfernt; nachher muss nach W. Kühne die Retina von der Papille abgeschnitten werden, was man mit einem kleinen Locheisen macht; noch einfacher kann man die ganze Papille mit dem N. opticus mittelst einer feinen Scheere herausschneiden. Dann legt man die Bulbushälfte in ein Schälchen mit $3 / 4 \% \mathrm{NaCl-Lösung} \mathrm{oder}$ mit schwacher Formollösung; durch leichtes Schütteln und Ziehen mit einer Pincette lässt sich die Netzhaut unter der Flüssigkeit ganz leicht von den anderen Häuten abziehen. Die isolirte Netzhaut wird dann in's Helle gebracht und angesehen. Das letztere muss möglichst rasch ausgeführt wer-

I) Die Enucleation am lebenden Kaninchen wurde ohne Cocainanästhesie gemacht, da Cocain nach $O$ vi o für den Sehpurpur nicht vollständig indifferent sein soll. Ovi o, Di una speciale azione della cocaina sulla funzione visiva. Annali di Ottalm. XXIV. suppl. dal Fasc. 4. p. 23. (1895.) 
Ueber das Verhalten des Sehpurpurs bei der Netzhautablösung. 423

den, da beim Tages- und besonders beim Sonnenlicht die Intensität der Farbe sehr rasch abnimmt.

Ich gehe jetzt zur näheren Beschreibung der von mir angestellten Versuche und der Resultate derselben über.

Schon die ersten Versuche stellten die Thatsache fest, dass sich die eigenthümliche Färbung der Stäbchenschicht auch an der abgelösten Netzhaut constatiren lässt. Die Anordnung dieser Versuche war folgende: Nach der Einführung des Fremdkörpers wurden die Kaninchen bei gewöhnlicher, schwacher Zimmerbeleuchtung gehalten ${ }^{1}$ ). Täglich wurde eine ophthalmoskopische Untersuchung bei gewöhnlichem Gaslicht vorgenommen. Einige Tage nach der Entwicklung der Netzhautablösung wurden die Augen bei derselben Zimmerbeleuchtung enucleirt, im Dunkeln in $10 \%$ Formollösung gehärtet und am folgenden Tag bei Natronlicht aufgeschnitten; alsdann wurden die Netzhäute bei Tageslicht angesehen. Sechs auf diese Weise angestellte Versuche ergaben Folgendes: in einem Fall mit 36stündiger Dauer der Ablösung waren die abgelösten Parthieen gut roth gefärbt, wäbrend die nicht abgelösten stark gelockert und mit der Chorioidea verklebt waren; im zweiten Falle waren zwei Tage nach der Entstehung der Netzhautablösung sowohl die abgelösten, als auch die nicht abgelösten Parthieen fast gleich stark blass-roth gefärbt, bei ziemlich gut erhaltenen Stäbchen, während in einem dritten Fall unter fast gleichen Bedingungen die Stäbchenschicht der abgelösten Theile orangegelb und die der nicht abgelösten purpurn gefärbt war. Im vierten und im fünften Fall, wo die Ablösung drei Tage bestanden hatte, waren die abgelösten Theile stellenweise

1) Die Käfige befanden sich in einem $3,25 \mathrm{~m}$ breiten, $5,0 \mathrm{~m}$ langen und $3 \mathrm{~m}$ hohen Zimmer des Erdgeschosses, welches ein 1,20 m: $1 \mathrm{~m}$ grosses Fenster nach Süden hatte. Die Käfige wurden vor directer Einwirkung des durch das Fenster fallenden Lichtes geschützt und erhielten nur von den Wänden reflectirtes Licht. 
gelb, stellenweise orange-gelb, bei vollständiger varicöser Degeneration der Stäbchenaussenglieder, während die nicht abgelösten schwach purpurn waren. Endlich im sechsten Fall, wo die Ablösung schon vier Tage alt war, konnte man an den abgelösten Stellen, wo die varicös-degenerirte Stäbchenschicht noch erhalten war, eine schwache gelbliche Färbung, und an den nicht abgelösten Stellen ziemlich gute Purpurfarbe constatiren.

Es wurden also an derabgelösten Netzhaut theils Sehpurpur, theils Zersetzungsproducte desselben constatirt. Da diese ersten Versuche noch nicht genïgend waren, um die Frage zu entscheiden, ob diese Zersetzung durch die Degeneration der Stäbchenschicht oder durch Lichteinwirkung bedingt war, so wurden noch weitere vergleichende Versuche angestellt, wobei die Kaninchen vor der Einwirkung des Tageslichtes geschützt und nur täglich einer kurzdauernden ophthalmoskopischen Untersuchung bei Gas- oder Natronlicht unterzogen wurden.

Die z weite Gruppe der Versuche wurde also in folgender Weise angestellt: nach der Einführung der Fremdkörper befanden sich die Kaninchen in einem dunkeln Käfig; die tägliche ophthalmoskopische Untersuchung, welche für jedes Auge einige Minuten dauerte, wurde im dunkeln Zimmer bei einer gewöhnlichen Gaslampe gemacht. Einige Tage nach der Entstehung der Ablösung wurden die entsprechenden Augen bei Natronlicht enucleirt. Die Enucleation wurde nicht sofort nach der letzten ophthalmoskopischen Untersuchung vorgenommen, sondern die Kaninchen unmittelbar vor der Enucleation mindestens 1 Stunde im Dunkeln gehalten. Nach der Härtung in Formol wurden die Augen am nächsten Tag bei Natronlicht präparirt und auf ihren Purpurbestand angesehen. Die Resultate dieser Versuche waren folgende: In 8 von 12 Versuchen wurden die abgelösten Theile der Netzhaut gefärbt gefunden, und zwar konnte man bemerken, dass die Farbe, je nach der Dauer 
der Ablösung, bald den ersten, bald den letzten farbigen Zersetzungsproducten des Sehpurpurs entsprach. So wurden z. B. die $12-24$ Stunden nach der Entstehung der Ablösung untersuchten Netzhäute ziemlich gut roth gefärbt gefunden; bei 2-21/2 tägiger Dauer der Ablösung war die Farbe in zwei Fällen blassroth und in zwei anderen orange oder intensiv gelb. Eine drei Tage nach der Entstehung der Ablösung untersuchte abgelöste Netzhautparthie war auch deutlich gelb; bei viertägiger Dauer der Ablösung endlich liess sich nur noch eine gelbliche Färbung constatiren. In allen diesen Fällen waren die nicht abgelösten Parthieen deutlich purpurn, oder wenigstens roth gefärbt; nur in einem Fall war die nicht abgelöste Parthie vollständig gelockert, so dass von irgend welcher Färbung keine Rede sein konnte. Was jetzt den Zustand der Stäbchenschicht anbelangt, so wurden die Stäbchen nur in zwei Fällen verhältnissmässig gut erhalten gefunden, d. h. man konnte die äusseren Glieder von den inneren unterscheiden; in allen anderen waren die äusseren Glieder varicös degenerirt und an manchen Stellen durch eine körnige Masse ersetzt, in welcher keine Spur der normalen Stäbchenstructur mehr zu erkennen war. Dabei entsprachen die degenerativen Erscheinungen weder, der Dauer der Ablösung, noch der Abnahme der Farbe an der abgelösten Netzhaut: ziemlich gut erhaltene Stäbchen waren einmal roth gefärbt, einmal gelblich, und umgekehrt zeigten in einem Fall körnig zerfallene Stäbchen ziemlich gute rothe Farbe. Die degenerativen und entzündlichen Erscheinungen der anderen Netzhautschichten waren verschieden ausgesprochen, aber anch sie standen in keiner directen Beziehung zum Schwinden des Sehpurpurs in der abgelösten Netzhaut.

In vier anderen Versuchen dieser Gruppe, bei welchen die abgelösten Netzhäute sechs, resp. zehn 'Tage nach der' Entstehung der Ablösung zur Untersuchung kamen, war gar keine Farbe mehr zu sehen. Die Ablösung war in allen diesen 
Fällen schon total und die mikroskopische Untersuchung zeigte eine ausgesprochene Degeneration des Netzhautgewebes; von der Stäbchenschicht waren nur in einem Fall (sechs Tage nach der Entstehung der Ablösung) structurlose Reste zu sehen, in den anderen Fällen fehiten dieselben vollständig.

In einer dritten Gruppe von Versuchen wurden die Kaninchen mit Netzhautablösung nicht nur vor dem Tageslicht, sondern auch vor dem Gaslicht geschützt. Während der ganzen Versuchszeit befanden sich die Thiere im dunkeln Käfig, und die tägliche ophthalmoskopische Untersuchung wurde bei Natronlicht vorgenommen. Die Enucleation der Augen und alle anderen Manipulationen mit ihnen wurden bei Natronlicht gemacht; nur auf ihren Purpurbestand wurden die Netzhäute bei Tageslicht untersucht. Die Netzhautablösung wurde nach verschiedenen Methoden hervorgerufen. In einem Fall wurde dieselbe durch Einführung eines Fremdkörpers aus Kupfer erzengt; nach 6 tägigem Bestehen der Ablösung wurde an der abgelösten Hälfte eine fleckige blass-rothe Färbung constatirt und die mikroskopische Untersuchung zeigte, dass die Stäbchenschicht nur an einigen Stellen in Form von Körnerhäufchen an der Aussenfläche der Netzhaut zurück geblieben war; die unabgelöste Hälfte, die ziemlich gut erhaltene Structur besass, war purpurn gefärbt. Im zweiten Fall, wo eine partielle Ablösung durch Einspritzung $\mathbf{3} / \mathbf{4} / 0$ NaCl-Lösung hervorgerufen war, wurde am neunten Tage nach dem Beginn der Netzhautablösung die abgelöste Parthie stäbchenlos gefunden, wobei von einer Färbung keine Rede war; die nicht abgelöste dagegen behielt gute Purpurfarbe. In acht anderen Versuchen, wo die Einspritzung der $3 / 4 \%$ NaCl-Lösung unter erhöhtem Druck vollzogen wurde und die Netzhaut gleich in ihrer ganzen Ausdehnung von der Chorioidea sich ablöste, fand ich folgendes Verhalten: 2 Stunden nach der Eintstehung der Ablösung (1 Versuch) war die 
Ueber das Verhalten des Sehpurpurs bei der Netzhautablösung. 427

Netzhaut noch intensiv roth, und die Stäbchen vollkommen gut erhalten; nach 1 Tag (1 Versuch) und nach 3 Tagen (2 Versuche) war die Farbe anch recht roth, wobei die Stäbchenaussenglieder nur in einem. Fall verhältnissmässig gut erhalten, im anderen dagegen varicös degenerirt und stellenweise in eine structurlose Masse zusammengeflossen waren; nach 6 Tagen (2 Versuche) waren die Netzhäute schon stellenweise stäbchenlos, an den Stellen aber, wo die Stächenschicht in Form von körnigen oder homogenen Häufchen zurückgeblieben war, war auch eine fleckige blassrothe Färbung zu sehen. Die nach 9 Tagen untersuchten Netzhäute (2Versuche) hatten schon keine Reste der Stäbchenschicht mehr und waren vollständig farblos.

Um eine Uebersicht über alle gemachten Versuche zu geben, füge ich eine Tabelle hinzu, durch welche die Färbung der abgelösten Netzhaut bei Einwirkung verschiedenen Lichtes, und zugleich das Verhältniss der Färbung zu der Zeit nach der Entstehung der Netzhautablösung veranschaulicht wird.

\begin{tabular}{|c|c|c|c|}
\hline $\begin{array}{l}\text { Zeit nach der } \\
\text { Entstehung der } \\
\text { Netzhaut- } \\
\text { ablösung }\end{array}$ & $\begin{array}{l}\text { Aufenthalt bei schwa- } \\
\text { cher Zinnmerbeleuch- } \\
\text { tung, ophthalmoskop. } \\
\text { Untersuchung beì } \\
\text { Gaslicht, täglich }\end{array}$ & $\begin{array}{c}\text { Aufenthalt } \\
\text { im Dunkeln, ophtbal- } \\
\text { moskop. Untersuchung } \\
\text { bei Gaslicht, } \\
\text { täglich }\end{array}$ & $\begin{array}{c}\text { Aufenthalt } \\
\text { im Dunkeln, ophthal- } \\
\text { moskop. Untersuchung } \\
\text { bei Natronicht, } \\
\text { täglich }\end{array}$ \\
\hline 2-12 Stunden & - & roth & roth \\
\hline 1. Tag & roth & roth & roth \\
\hline 2. $"$ & $\begin{array}{c}\text { blass-roth oder } \\
\text { orange }\end{array}$ & $\begin{array}{c}\text { blass-roth oder } \\
\text { orange }\end{array}$ & - \\
\hline 3. " & orange oder gelb & $\begin{array}{l}\text { blass-roth, orange, } \\
\text { oder gelb }\end{array}$ & roth \\
\hline & blass-gelb & gelb & - \\
\hline 5. & - & 一 & - \\
\hline $6 . \quad "$ & - & farblos & blass-roth \\
\hline $\left.\begin{array}{l}\text { 7. Tag und } \\
\text { später }\end{array}\right\}$ & - Vollkommene & Fehlen der Stä & henschicht. \\
\hline
\end{tabular}

Alle oben beschriebenen Versuche haben also ergeben, dass sich die Färbung der äusseren Schicht der abgelösten v. Graefe's Arehiv für Ophthalmologie. XLIV. 
Netzhaut mit Sehpurpur oder mit den farbigen Zersetzungsproducten desselben unter gewissen Umständen constatiren lässt, so lange noch die A ussenglieder der Stäbchen unverändert oder wenigstens in zerfallenem $Z u$ stande vorhanden sind, $d . h$. bis zum 6 . oder 7 . Tage vom Beginn der Ablösung der Netzhaut bei Experimenten an Kaninchen. Weiter zeigten die Versuche, dass die degenerativen und entzündlichen Processe in der abgelösten Netzhaut, insbesondere die in der Stäbchenschicht, an und für sich keinen Einfluss auf die Färbung der letzteren haben; zwei Netzhäute, eine im Besitz einer fast normalen Stäbchenschicht, die andere mit structurlosem Zerfall derselben, können, wie aus Obigem hervorgeht, unter gewissen Umständen gleich gefärbt sein. Als die Ursache der Farbenveränderungen des Sehpurpurs und des allmählichen Schwundes desselben in der abgelösten Retina ist also die Lichteinwirkung anzusehen. Wird ein Kaninchen mit Netzhautablösung vor dem Tages- und Gaslicht geschützt und nur täglicher kurzdauernder Belichtung mit dem schwach auf den Sehpurpur wirkenden Natronlicht ausgesetzt, so kann man sogar am sechsten Tage, d. h. bis zum Ende des Vorhandenseins der Stäbchenschicht, nur eine Abschwächung der Purpurfarbe bis zu blassroth constatiren; wird dagegen das Thier bei gewöhnlicher Zimmerbeleuchtung oder auch im Dunkeln gehalten, aber dabei täglicher Belichtung mit dem Ophthalmoskop bei einer Gaslampe unterzogen, so bleicht die Farbe der Netzhaut stufenweise in einigen (etwa vier) Tagen, bis zur völligen Farblosigkeit aus.

Nachdem wir diese Thatsachen constatirt haben, wollen wir jetzt zur Betrachtung des Wesens der Processe iubergehen, auf welchen die Färbung der abgelösten Netzhaut beruht.

Wenn wir die Netzhaut eines enucleirten purpurhaltigen Auges von der Chorioidea und von der an letzterer liegen- 
Ueber das Verhalten des Sehpurpurs bei der Netzhautablösung. 429

bleibenden Epithelschicht abziehen, so ruft diese Manipulation an und für sich, wie bekannt, keine Zerstörung der Farbe der Netzhaut hervor; eine solche isolirte Netzhaut kann ihres Purpurs nur durch Licht beraubt werden. Es unterliegt also keinem $Z$ weifel, dass auch bei der Entstehung der Ablösung in einem lebenden Auge die Netzhant mit dem ganzen Vorrath an Sehpurpur, welcher unmittelbar vor dem Moment der Ablösung in ihrer Stäbchenschicht gebildet oder vorhanden war, - abgelöst wird. Beobachten wir also eine Purpurfärbung der Netzhaut in den ersten Momenten nach der Entstehung der Ablösung; so haben wir dabei sicher den zurückgebliebenen Sehpurpur vor uns. Wenn wir aber eine solche Färbung, obschon von abnehmender Intensität, auch nach Einwirkung irgend welcher Beleuchtung auf das Auge, ziemlich lange Zeit hindurch zur Beobachtung bekommen, so könnten wir dieses Vorkommen des Sehpurpurs in der abgelösten Retina auf zweierlei Weise erklären:

1. In der abgelösten Netzhaut kommt nicht ausschliesslich die Zersetzung des Sehpurpurs vor, sondern auch eine selbständige Regeneration desselben aus seinen Zersetzungs producten; wenn bei längerem Aufenthalt des Thieres bei einer gewissen Beleuchtung eine allmähliche Abschwächung der Purpurfarbe $\mathrm{zu}$ beobachten ist, so handelt es sich nur darum, dass bei dieser Beleuchtungsintensität der Process der Zersetzung über den der Regeneration prävalirt.

2. In der abgelösten Netzhaut findet keine Regeneration statt; wenn der Vorgang der Bleichung des Sehpurpurs sich über einige Tage erștreckt, so ist die Ursache dieser Erscheinung nur in der Schwäche der betreffenden Beleuchtung zu suchen.

Um für eine von diesen zwei Erklärungen die Entscheidung treffen zu können, musste man natïrlich auf experimentellem Wege die Möglichkeit der Regeneration an der abgelösten Netzhaut nachzuweisen versuchen. 
$\mathrm{Zu}$ diesem $\mathrm{Z}$ weck wurden zuerst Versuche mit isolirt gebleichten Netzhäuten angestellt und zwar in folgender Weise. $Z u$ den Versuchen dienten Augen mit Netzhautablösung, welche durch Einführung von Fremdkörpern aus Kupfer hervorgerufen war, in welchen die Daner der $\mathrm{Ab}$ lösung zwei Tage nicht überschritt, und die abgelösten Parthieen noch roth oder blassroth gefärbt blieben. In einem Fall wurde die einem solchen Auge entnommene Netzhaut den directen Sonnenstrablen ausgesetzt und, nach völliger Ausbleichung während einiger Minuten, in einer feuchten Kammer ausgebreitet wieder in's Dunkle gebracht. Nach zwei Stunden wurde dieselbe bei schwachem Tageslicht wieder flüchtig angesehen und noch farblos gefunden; wieder in's Dunkle gebracht, verblieb sie auch bis zum nächsten Tag, ohne eine Spur von Wiederfärbung zu zeigen. Vier andere Versuche wurden so angestellt, dass die abgelösten Netzhautparthieen, nach völliger Ausbleichung beim Sonnenlicht, wieder sorgfältig auf der Oberfläche der Chorioidea ausgebreitet und in feuchter Kammer in's Dunkle gebracht wurden. Trotz dieser Berührung der Netzhant mit der Epithelschicht und trotz langem Verweilen (ca. 20 bis 24 Stunden) im Dunkeln kehrte die Färbung niemals wieder.

Für die Erklärung des negativen Befundes dieser Versuche-wollen wir uns zuerst an die von W. Kühne aufgestellte These erinaern, dass für die epitheliale Regeneration des Sehpurpurs ein lebender, oder wenigstens überlebender Zustand des Epithels durchaus nothwendig ist; im herausgenommenen Auge vom Kaninchen verliert aber, wie bekannt, das Epithel sofort seine Lebenseigenschaften. Aber auch eine isolirte gebleichte Kaninchennetzhaut ist, nach W. Kühne, bei längerem (wenigstens zweibis dreistündigem) Verweilen im Dunkeln einer partiellen Regeneration des Sehpurpurs aus seinen Zersetzungsproducten fähig, - es kommt ihr die sogen. Autoregeneration zu, ver- 
möge des in der Stäbchenschicht zurückgebliebenen Rhodophylins. In unseren Versuchen wurde die Netzhaut lange Zeit im Dunkeln gehalten, - isolirt oder in Berührung mit der Epithelschicht, das ist ganz gleich, - sie befand sich somit unter Bedingungen, welche eine Autoregeneration ermöglicht hätten. Da dieselbe nicht zum Vorschein kam, so könnte man vielleicht hier, der Theorie Kühne's folgend, annehmen, dass die am lebenden Thiere abgelöste Netzhaut nur ihren Purpur, aber nicht das Rhodophylin behält.

Aus den Versuchen an der dem Auge entnommenen Netzhaut des Kaninchens kann man sich aber keine unbestreitbare Meinung über die Fähigkeit der abgelösten Netzhaut zur Regeneration des Sehpurpurs im lebenden Kaninchenauge bilden. Die Versuche über die Autoregeneration gelingen selbst an der normalen dem Auge entnommenen Netzhaut des Kaninchens nicht in allen Fällen und geben auch keine so deutlichen Resultate, dass man sicher erwarten könnte, die Autoregeneration bei einer veränderten und destruirten Netzhaut zu beobachten. Von zehn zu diesem Zwecke bei normalen Kaninchenaugen angestellten Controlversuchen gelang es mir nur in zwei Fällen, etwas der Autoregeneration ähnliches $\mathrm{zu}$ beobachten. Ich bemühte mich daher, Versuche an lebenden Thieren anzustellen, um mich der Lösung dieser Frage zu nähern.

$\mathrm{Zu}$ letzterem $\mathrm{Zwecke} \mathrm{benutzte}$ ich zuerst Kaninchen, bei welchen die Netzhautablösung durch die Einführung von Fremdkörpern aus Kupfer hervorgerufen wurde. Die Kaninchen befanden sich im dunkeln Käfig und wurden einige Male bei Gaslicht ophthalmoskopirt. Die Versuche wurden am ersten oder spätestens am zweiten Tage nach der Entstehung der Ablösung vorgenommen, und bestanden darin, dass die betreffenden Kaninchen mit atropinisirten Augen starker Beleuchtung ausgesetzt und nachher für zwei bis 
drei Stunden wieder in's Dunkle gebracht wurden; diese Zeit ist für ein Kaninchenauge mehr als genügend, um den der normalen Netzhaut entzogenen Sehpurpur wieder herzustellen. Danach wurden die Augen bei Natronlicht enucleirt und nach gewöhnlicher Formolhärtung der Inspection bei Tageslicht unterworfen. In zwei Versuchen, wo die Netzhautablösung total war, und die Besonnung eine Stunde dauerte ${ }^{1}$ ), wurde trotz dreistündigem Verweilen des Kaninchens im DunkeIn keine Färbung der äusseren Fläche der Netzhaut beobachtet. Die mikroskopische Untersuchung zeigte, dass in beiden Fällen die Stäbchenschicht erhalten und die Stäbchenaussenglieder nur stellenweise durch körnige Masse ersetzt waren; es lag also die Ursache des negativen Befundes nicht im Fehlen der Stäbchenschicht. In drei anderen Versuchen wurden Augen mit partieller Netzhautablösung eben solcher Besonnung unterzogen; während nachträgliches zwei- bis dreistündiges Verweilen im Dunkeln vollständig genügend war, um die Farbe der nicht abgelösten Parthieen bis zum Sehpurpur zu regeneriren, blieben die abgelösten Parthieen vollständig farblos. Die mikroskopische Untersuchung ergab auch hier, dass die abgelösten Parthieen ihre Stäbchenschicht, obschon mehr oder weniger degenerirt, noch besassen. Ein auf den ersten Blick in Widerspruch mit den beschriebenen Versuchen stehendes Resultat ergaben folgende zwei Versuche, bei welchen die abgelösten Parthieen der Netzhaut nach starker Belichtung und nachträglichem Verbleiben der Kaninchen im Dunkeln gelb gefärbt gefunden wurden. Es handelte sich aber nur um eine unvollständige Bleichung, nicht um eine Regeneration des Sehpurpurs: so hatte z. B. in einem von diesen Versuchen die Besonnung nur eine halbe

1) Damit die Kaninchen ihren Platz in Bezug auf die Lichtquelle beibehielten, wurden sie in sogen. Würzburger Kaninchenhalter eingesperrt und das entsprechende Auge mittelst eines Sperr-Elevateurs offen gehalten. 
Stunde gedauert, was bei dem Vorhandensein feiner Trübungen in Augen mit Netzhautablösung augenscheinlich nicht genügend ist, um eine völlige Bleichung des Sehpurpurs herbeizuführen, und in dem anderen Fall wurde das Auge, in Ermangelung des Sonnenscheines am Versuchstage, nicht den Sonnenstrahlen, sondern eine halbe Stunde lang dem Licht eines Auer'schen Brenners ausgesetzt. Versuche mit Besonnung an denjenigen Augen, an welchen die Netzhautablösung durch Einspritzung von physiologischer Kochsalzlösung unter erhöhtem Druck hervorgerufen wurde, und welche dichte Trübungen des Glaskörpers aufwiesen, zeigten, dass sogar zweistündige Besonnung nicht genügend ist, um eine vollständige Bleichung des Sehpurpurs zu bewirken. Besonders instructiv war folgender Versuch, welcher gleichzeitig an drei Kaninchen vorgenommen wurde: I-normales Kaninchen, II und IIIKaninchen, welchen man drei Tage vorher Einspritzungen von $3 / 4 \%$ iger $\mathrm{NaCl}$-Lösung in den Glaskörper gemacht hatte; an allen vier Augen der letzten beiden Kaninchen hatten sich dichte Trübungen im Glaskörper entwickelt. Die linken Augen aller Kaninchen wurden zugebunden, während die rechten alle gleichzeitig der Besonnung ausgesetzt wurden. Nach 21/4 stündiger Besonnung wurden alle rechten Augen schnell enucleirt. Hierauf wurden die. linken Augen für $2{ }^{1} / 4$ Stunden derselben Beleuchtung ausgesetzt. Alsdann wurden die Kaninchen 18 Stunden hindurch in's Dunkle gebracht, und nach Verlauf dieser Zeit auch die linken Augen enucleirt. Die Untersuchung der in Formol gehärteten Augen zeigte Folgendes: die Netzhaut des rechten Auges von Kaninchen I vollständig farblos, die von II und III total abgelöst und gelblich gefärbt. Es wurde also als unmittelbares Resultat der Besonnung constatirt, dass dieselbe genügend war, um einer normalen Netzhaut den Sehpurpur völlig zu rauben, dass sie aber an den beiden Augen mit Netzhautablösung wegen der Glas- 
körpertrübungen nicht ganz dieselbe Wirkung auszuüben vermochte. Wäre nun an den abgelösten Netzhäuten eine Regeneration möglich, so könnte man erwarten, an den beiden anderen Augen mit Netzhautablösung, welche sich nach derselben Beleuchtung noch 18 Stunden im Dunkeln befanden, eine wenn auch nicht völlige, so doch immerhin partielle Regeneration des Sehpurpurs zu finden. Es ergab sich aber das Gegentheil: während die Netzhaut des linken Auges des normalen Thieres gut purpurn gefunden wurde, waren die beiden abgelösten Netzhäute ebenso gelblich gefärbt wie die der sofort nach der Besonnung enucleirten Augen. Dass die Trübung der brechenden Medien verzögernd auf den Zersetzungsprocess des Sehpurpurs wirkt, zeigte auch ein zur Controle angestellter Versuch, in welchem zwei Augen von demselben Kaninchen, das eine mit ganz normalem Glaskörper und das zweite mit dichten undurchsichtigen Trübungen desselben in Folge von künstlich hervorgerufener Glaskörperblutung, gleicher Besonnung ausgesetzt wurden; während dieselbe genügend war, um den Sehpurpur des ersten Auges zur vollständigen Bleichung zu bringen, bewirkte sie keine Veränderung des Purpurs im zweiten Auge ${ }^{1}$ ).

So haben die Versuche gezeigt, dass die abgelöste Netzhaut des lebenden Auges, welche von ihrem regenerirenden Epithel getrennt ist, durch ihr innewohnende Kräfte nicht einmal eine partielle Regeneration des Sehpurpurs aus seinen Zersetzungsproducten bewirken kann. Dies lässt sich auch nach den Kühne'schen Theorieen in folgender Weise erklären: da die abgelöste Netzhant von dem Epithel

1) Hier kann ich nicht unterlassen, zu bemerken, dass die von W. Kühne zum Nachweis der Regenerationserscheinungen angegebene optographische Methode für unsere Versuche leider nicht anwendbar war. 
Ueber das Verhalten des Sehpurpurs bei der Netzhautablösung.

getrennt ist, so kann sie durch den Process der Neogenese den verlorenen Purpur nicht wieder erhalten; ausserdem aber wird dabei die abgelöste Retina an ihrer äusseren Seite durch eine grosse Menge von subretinaler Flüssigkeit bespiult, und man muss daher nach der Analogie mit den Versuchen Kühne's an curarisirten Fröschen annehmen, dass auch hier die Zersetzungsproducte des Sehpurpurs aus den Stäbchen ausgezogen werden; wenn dieselben aber in der Stäbchenschicht nicht mehr vorhanden sind, so sind auch keine anagenetischen Processe in derselben mehr möglich.

Auf Grund von allem oben Erwähnten kann die Langsamkeit des Bleichungsprocesses der abgelösten Netzhaut im Leben bei geringeren Be. leuchtungsintensitäten nur auf der schwachen Wirkung derselben auf den Sehpurpur beruhen. Den weiteren Beweis für letztere Annahme können wir auch durch Controlversuche mit Wirkung verschiedener Beleuchtung auf die Schnelligkeit der Bleichung des Purpurs erbringen.

In dieser Richtung wurden untersucht:

1. Die Wirkung schwacher Zimmerbeleuchtung und des Ophthalmoskopirens auf den Purpur eines lebenden normalen Kaninchenauges. Es ergab sich, dass die Netzhäute von Kaninchen, welche sich vor der Enucleation lange Zeit (resp. mehrere Tage) bei gewöhnlicher Zimmerbeleuchtung befanden, ebenso gut purpurhaltig sind, wie diejenigen von Dunkelthieren. Das Ophthalmoskopiren eines normalen Kaninchenauges bei Gasbeleuchtung von gewöhnlicher mittlerer Intensität während 30 bis 40 Minuten ruft auch noch keine erhebliche Bleichung des Purpurs hervor. Nur nach einer etwa 1 Stunde lang dauernder Belichtung dieser Art kann man einen Unterschied der Netzhautfarbe eines ophthalmoskopirten und nicht ophthalmoskopirten Auges bemerken. Bei den betreffenden 
Versuchen wurde natürlich darauf geachtet, die Augen sofort nach einer derartigen Belichtung zu enucleiren, sie halbirt ohne Verweilen in $10 \%$ Formollösung einzuwerfen und in's Dunkle zu bringen; dabei wurden durch diese Härtung alle denkbaren Regenerationsprocesse ausgeschlossen und die vorhandene Färbung nicht geschädigt. Die scheinbare Indolenz des Sehpurpurs gegen die gewöhnliche Zimmerbeleuchtung und, theilweise, auch gegen die ophthalmoskopische Beleuchtung innerhalb gewisser Zeit können wir also, in Uebereinstimmung mit Kühne, durch einen Gleichgewichtszustand der zu gleicher Zeit in der Retina stattfindenden Zersetzungs- und Regenerationsprocesse erklären.

2. Wirkung der oben erwähnten Beleuchtungseinflüsse auf die Netzhaut von enucleirten unaufgeschnittenen Kaninchenaugem. Für die Versuche wurde eine gewisse Anzahl von zu gleicher Zeit enucleirten Augen von Dunkelkaninchen benutzt; eine Hälfte davon wurde feucht im Dunkeln gehalten, die anderen, ebenfalls feucht, in den Käfig gestellt, worin sich gewöhnlich die Kaninchen befanden. Die letzteren Augen wurden täglich durch eine Gaslampe ophthalmoskopisch beleuchtet, (jedes Auge fünf Minuten lang). Nach gewissen Zeiträumen wurde je eines von denjenigen Augen, welche sich im Dunkeln und bei schwacher Zimmerbeleuchtung befanden, genommen und, nach zwei- bis dreistündiger Härtung in 10\% Formollösung im Dunkeln, auf seinen Purpurbestand untersucht.

Die Resultate waren folgende:

Nach einem Tag war die Dunkelnetzhaut gut purpurn, die belichtete gut purpurn.

Nach zwei Tagen war die Dunkelnetzhaut gut purpurn, die belichtete blass-roth.

Nach drei Tagen war die Dunkelnetzhaut gut purpurn, die belichtete gelb.

Es waren an beiden Netzhäuten bereits Macerationserscheinungen $\mathrm{zu}$ bemerken. 
Ueber das Verhalten des Sehpurpurs bei der Netzhautablösung. 437

Nach vier Tagen und später waren die inneren Häute beider Augen vollständig macerirt und mit dem aufgelösten Chorioidealpigment durchsetzt, so dass trotz der nachträglichen Formolhärtung keine Purpurfärbung zu erkennen war.

Es wurde also bei diesem Versuch eine langsame, sich auf drei bis vier Tage erstreckende Bleichung des Sehpurpurs constatirt. Da in einem enucleirten Kaninchenauge schon einige Momente nach dem Tode alle im $\mathrm{Zu}$ sammenhang mit den Lebenseigenschaften des Pigmentepithels stehenden Regenerationsprocesse als vollständig ausgeschlossen anzusehen sind, so vollzieht sich hier die Bleichung des Purpurs proportional der Zeit der Lichteinwirkung von gleicher Intensität. Erinnern wir uns jetzt, dass die Bleichung der abgelösten Netzhaut in einem lebenden Kaninchenauge, bei derselben Beleuchtung wie bei diesem Versuch, gerade so in drei bis vier Tagen allmählich verläuft, so bekommen wir durch diesen Vergleich eine wichtige Bestätigung unserer Ansicht, dass die abgelöste lebende Netzhaut sich von einer todten Netzhaut, in Bezug auf die Regenerationslosigkeit, in keiner Weise unterscheidet.

3. Um zu entscheiden, ob bei obigen Versuchen die schwache Zimmerbeleuchtung oder die Belichtung bei dem Ophthalmoskopiren hauptsächlich die Bleichung des Purpurs der abgelösten Netzhaut bewirkte, wurden Controlversuche über die Wirkung dieser Lichteinflüsse auf die isolirte Kaninchennetzhaut angestellt. Es wurde dabei beobachtet, dass eine purpurn entnommene frische Netzhaut bei schwacher Zimmerbeleuchtung (feucht in den Kaninchenkäfig gestellt) sehr langsam bleicht: nach zwei Stunden bleibt sie noch gut purpurn, nach 18 Stunden wird sie blass-roth, nach zwei Tagen gelb, und erst nach $2 \%$ Tagen farblos. Wenn wir jetzt noch in Betracht ziehen, dass eine isolirte Netzhaut, caeteris paribus, viel mehr belichtet wird, als eine 
abgelöste Parthie im Inneren des Auges, welche das Licht nur durch die Pupille bekommt, so kann es nicht überraschen, dass der Process der Bleichung in letzterem Fall noch langsamer (in drei bis vier Tagen, wie oben erwähnt) verläuft. Viel intensiver wirkt auf den Purpur die ophthalmoskopische Beleuchtung: nach 15 Minuten solcher Belichtung wird eine purpurne Netzhaut blass-roth, nach 50 Minuten gelb, nach 1 Stunde bis 1 Stunde 20 Minuten blass-gelb und nach etwa 1 Stunde 30 Minuten farblos. So können wir uns vorstellen, dass jede einige Minuten dauernde ophthalmoskopische Beleuchtung eine gewisse Menge von Purpur der abgelösten Netzhaut auch im lebenden Auge zur Zersetzung bringt, vielleicht in höherem Maasse, als dies einen Tag hindurch dauernde schwache Zimmerbeleuchtung zu bewirken vermag. Summirt man die Wirkung wiederholter ophthalmoskopischer Untersuchungen auf den Sehpurpur, so bekommt man schon eine erhebliche Bleichung desselben. Ziehen wir dies alles in Betracht, so können wir daraus leicht erklären, warum die Resultate der Versuche der ersten und zweiten Gruppe (siehe die.Tabelle) in Bezug auf die Bleichungszeit des Purpurs keinen grossen Unterschied zeigen, obschon in dem einen Fall die Kaninchen vor dem Tageslicht geschützt waren, in dem anderen nicht: es war dabei die ophthalmoskopische Untersuchung der wichtigste Bleichungsfactor, nicht das schwache Tageslicht.

4. Die Untersuchung über die Wirkung des Natronlichtes auf eine isolirte Netzhaut zeigte, dass dieselbe stundenlang bei dieser Beleuchtung ohne erhebliche Abschwächung der Farbe liegen kann. In Folge dessen können wir mit einem gewissen Recht die Abschwächung der Purpurfarbe an den abgelösten Netzhäuten der Dunkelthiere (siehe die Tabelle) gewissermaassen auf Resorption des Sehpurpurs, wie es auch W. Kühne für die curarisirten Frösche als möglich annimmt, zuriickfïhren. Fine weitere und genauere Ver- 
Ueber das Verbalten des Sehpurpurs bei der Netzhautablösung. 439

folgung dieser Erscheinung, zum, Zweck der Bestätigung dieser Anschauung, lässt sich aber experimentell nicht ausführen, in Folge der bald stattfindenden vollständigen Zerstörung der Stäbchenschicht.

Ich gestatte mir jetzt, die erlangten Resultate dieser Arbeit zusammenzustellen.

1. Die Färbung der äusseren Schicht der Netzhaut mit dem Sehpurpur oder mit den farbigen Zersetzungsproducten desselben lässt sich, unter gewissen Umständen, auch an der abgelösten Netzhaut constatiren.

2. Bei experimentell erzeugter Netzhautablösung an Kaninchen wird diese Erscheinung nur bis zum sechsten bis siebenten Tage nach dem Beginn der Ablösung beobachtet, d. h. so lange noch die Aussenglieder der Stäbchen unverändert oder wenigstens in zerfallenem Zustande vorhanden sind.

3. Die Färbung der abgelösten Netzhaut hängt, bei Ausschluss des Tichtes, lediglich von dem Vorrath an Sehpurpur $a b$, welcher im Momente der Ablösung in der Stäbchenschicht vorhanden ist und zu der Zeit gebildet wurde, wo die Aussenglieder der Stäbchen von der Epithelschicht der Retina noch nicht abgelöst waren.

4. Die degenerativen und entziindlichen Processe in der abgelösten Netzhaut, insbesondere die in der Stäbchenschicht, haben keinen Finfluss auf die Färbung der letzteren: zwei Netzhäute, eine im Besitz einer normalen Stäbchenschicht, die andere mit structurlosem Zerfall derselben, können unter gewissen Umständen gleich gefärbt sein.

5. Der Purpur der abgelösten Netzhaut unterliegt nur dem Vorgang der Zersetzung; eine Regeneration des zersetzten Purpurs ist in derselben nicht zu beobachten.

6. Als Factor, welcher die Zersetzung des Sehpurpurs in der abgelösten Retina und die damit verbundenen Farbenveränderungen der Stäbchenschicht bewirkt, ist das Licht zu betrachten. 
7. Die Schnelligkeit des Zersetzungsprocesses des Sehpurpurs hängt direct von der Intensität des in das Auge fallenden Lichtes $a b$, und nur Trübungen der brechenden Medien wirken, je nach ihrer Dichtigkeit, auf die Bleichung des Purpurs verzögernd.

8. Starke Beleuchtung mit directem Sonnenlicht bringt an einem Auge mit Netzhautablösung und feinen Glaskörpertrübungen den Sehpurpur der abgelösten Parthieen in $1 / 2-1$ Stunde zur völligen Bleichung; bei Vorhandensein dichter Glaskörpertrübungen bleibt sogar nach 21/4 stïndiger Besonnung noch eine blass-gelbliche Färbung an den abgelösten Parthieen bestehen.

9. Wird ein Thier mit Netzhautablösung bei schwacher Zimmerbeleuchtung oder im Dunkeln gehalten, aber täglich wiederholter ophthalmoskopischer Untersuchung unterworfen, so kommt es zu langsamer Bleichung der Farbe der abgelösten Theile, wobei der Sehpurpur im Laufe einiger (etwa vier) Tage, durch Roth, Blassroth, Orange, Gelb in Blassgelb und schliesslich in eine farblose Substanz (Sehweiss) übergeführt wird.

10. Wenn das Kaninchen immer im Dunkeln verbleibt und das Auge mit Netzhautablösung nur täglicher, kurzdauernder Belichtung mit dem schwach auf den Purpur wirkenden Natronlicht ausgesetzt wird, so kann man sogar am sechsten Tage nach der Entstehung der Ablösung, trotz völliger Degeneration der Stäbchenschicht, nur eine Abschwächung der Purpurfarbe bis zu Blassroth constatiren.

11. Eine theoretisch mögliche allmähliche Resorption des Sehpurpurs der abgelösten Netzhaut ohne jeden Lichtzutritt zum Auge lässt sich experimentell nicht mit Sicherheit constatiren, in Folge der bald stattfindenden Zerstörung der Stäbchenschicht.

12. Die Anschauungen W. Kühne's und seiner Schüler über das Wesen der Regenerations- und Zersetzungsprocesse des Sehpurpurs sind auch zur Erklärung aller bei 
Ueber das Verhalten des Sehpurpurs bei der Netzhautablösung. 441

der experimentellen Netzhautablösung zu beobachtenden Erscheinungen anwendbar; die letzteren Experimente bestätigen auch die Rolle des Epithels der Retina bei diesem Processe, als des einzigen activen Regenerators des Sehpurpurs.

Ich muss endlich noch die Frage berühren, inwieweit die Ergebnisse der Versuche über den Sehpurpur bei der Netzhautablösung des Kaninchens sich auf die Netzhautablösung beim Menschen übertragen lassen.

Ich habe schon früher bemerkt, dass die Veränderungen der Netzhaut und besonders ihrer Stäbchenschicht bei der Netzhautablösung des Menschen nicht so schnell und nicht so stark hervortreten wie beim Kaninchen; wie lange die Stäbchenschicht beim Menschen erhalten bleibt, darüber liegen in der Literatur keine directen Angaben vor. Es findet sich meistens nur die Gelegenheit zur Untersuchung solcher Augen, bei welchen die Netzhautablösung schon Jahre lang bestanden hat, und in welchen die Stäbchenschicht verschwunden oder zu tropfen- oder kolbenförmigen Gebilden degenerirt ist. Die seltenen Fälle aber, welche ziemlich frühzeitig zur Untersuchung gelangten, zeigen, dass dabei die Structur der Netzhaut wenigstens Wochen, wenn nicht Monate lang völlig gut erhalten bleiben kann. So beschreibt Nordenson ${ }^{1}$ ) einen Fall, in welchem die Netzhautablösung in Folge einer Geschwulst der Chorioidea sich entwickelt hatte, und wo die abgelöste Netzhaut $4 \% 2$ Wochen nach dem Beginn der Ablösung sich mikroskopisch völlig normal erwies.

Endlich spricht für die Erhaltung der Structur in einigen Fällen auch die Möglichkeit der Wiederherstellung des Sehvermögens nach geheilter Netzhautablösung.

Auf diese Erfahrungen gestützt kann man das positive Ergebniss der Versuche, dass die Färbung der abgelösten Netzhaut im Dunkeln oder bei schwacher Beleuchtung

1) L. cit. S. 164. 
442 N. Andogsky, Ueber das Verhalten des Sehpurpurs etc.

ziemlich lang erhalten bleibt, mit Sicherheit auf den Menschen iibertragen. Aber auch die negativen Befunde können, wie z. B. das Fehlen der Regeneration in der abgelösten Netzhaut, augenscheinlich auf den Menschen übertragen werden, da alle Versuche mit dem Sehpurpur zeigen, dass die Stäbchenschicht, sei sie verändert oder nicht, an und für sich keine active Rolle in diesem Processe spielen kann.

Was aber die Frage betrifft, ob nach völliger Heilung der Netzhautablösung beim Menschen auch die Regenerationsprocesse in der Netzhaut wieder hergestellt werden, so sind die Versuche, auf experimentellem Wege dieser Frage näher zu treten, in Folge technischer Schwierigkeiten gescheitert. Wenn wir aber diese Frage theoretisch betrachten, so ist die Annahme, dass eine Wiederherstellung der Neubildung des Sehpurpurs vorkomme, sehr wohl zulässig, aber nur in jenen Fällen, in welchen nicht nur die Structur der Stäbchenschicht, sondern auch die des Epithels vollständig oder wenigstens hinreichend gut erhalten geblieben ist.

Am Schlusse erfülle ich die angenehme Pflicht, Herrn Prof. Th. Leber für die Anregung und liebenswürdige Unterstützung bei Ausführung dieser Arbeit meinen verbindlichsten Dank auszusprechen. 